\title{
An Intimation System for Villages Surrounded by the Forest
}

\author{
Mukesh Kumar Singh, Sridhar N K
}

\begin{abstract}
The accessibility of deployed sensor networks from far distance increased day by day. If any animal intrusion and fire detection information detected form sensor nodes can be accessed by the administrator at the far distance from the boundary line of forest $(B L O F)$, in the form of meter to meter updates, then, it will be very beneficial for a given village area surrounded by the forest. The Internet of Things (IoT) based layered wireless sensor networks (IoT-BLWSNs) are the best solution for the above presented applications. The IoT-BLWSNs will also be applicable for restricted (hospital, industrial area, animal cage monitoring in zoo etc) and non restricted areas (college and school campus).

In the recent years for heterogeneous as well as homogenous layered networks data transmission have been carried out form layers to the cloud in the IoT-BLWSNs. Each sensor nodes within the layer will sense different events in heterogeneous layered networks. For the proposed heterogeneous based layered sensor networks system model, we consider the processing of the data from sensors to the destination will be carried out wirelessly. The received data will get stored into the cloud with the help of Microcontroller at the destination end. Further, a remotely controlled drone will be sent to the corresponding point based on the received location information from the administrator end for the visualization of the intruder. A reliable protection layer to the villagers and crops will get provided from meter to meter updates for animal intrusion and fire detection.
\end{abstract}

Key words: IoT-BLWSNs, BLOF, meter to meter update, intrusion detection, heterogeneous.

\section{INTRODUCTION}

One of the most significant developments in the area of WSNs is the cluster-based wireless sensor networks (CBWSNs). For more efficient resource utilization and lower energy consumption clustering allows sensors to efficiently coordinate their local interactions. From a large number of resource-constrained sensors a massive heterogeneous data will get generated and processed in the IoT based layered wireless sensor networks (LWSNs) due to its enormous storage and computing capabilities. Limitations of sensing and communication range for sensors can overcome in order to monitor a given large area with the help of cluster deployment of sensor nodes. Dependency on cluster head for computing and processing the data to the fusion center is the major drawback of cluster deployed sensor network. For the leach protocol all sensor nodes to be equally capable of computing and processing of data for the random selection of cluster head, also relay nodes required to make communication of data till the fusion center, which is also a

Revised Manuscript Received on July 05, 2019.

Dr.Mukesh Kumar Singh, Sri Venkateshwara College of Engineering, Bengaluru- 562157.

Mr.Sridhar N K Sri Venkateshwara College of Engineering, Bengaluru562157.

mkmukeshkumarsingh@gmail.com, sridharnk52@gmail.com. limitation for the clustered sensor networks. Hence, we require IoT based layer sensor networks in order to overcome the limitations of the cluster sensor networks.

The purpose of our work is to formulate a problem for the development of the IoT-BLWSNs. In order to make presented system topology more suitable for real time applications we need to develop IoT-BLWSNs, where each layer will be having a finite number of heterogeneous sensor nodes.

The proposed IOT based clustered WSN will be useful in the application where administrator will be at very far distance from deployed network such as village surrounded by forest. The presented work will also be useful in restricted campus area, where different events have to be detected with priority basis such as, in the hospital, ICU unit, Ward unit, and Emergency unit etc to be accessed by a common administrator. The given system model can also be used for monitoring the animal cage in the zoo campus. Further, the proposed network can be used for non restricted campus area where administrator wants to access the undesired detected events form any corner of the notified area.

\section{LITERATURE SURVEY}

The power allocation, node-positioning and reliability for cluster based WSNs of different topologies had carried out in M. K. Singh, G. Sharma and N. Naik (2014). Cattle monitoring with the help of $\mathrm{Wi}-\mathrm{Fi}$ and ZigBee for heterogeneous wireless network has been proposed in [1]. The work in [2] has proposed traffic monitoring with the help of vehicular sensor network. In the battlefield surveillance system, distributed parameter estimation has been presented based on cloud computing for mobile wireless sensor network in [3]. In [4], vehicle location system and monitoring for citizen safety has been proposed using wireless sensor network. A three-layer low-energy adaptive clustering hierarchy has been presented for wireless sensor networks in [5]. In order to improve lifetime of heterogeneous wireless sensor networks multi-layer realization has been carried out in [6]. The design for public safety network has been proposed in [7]. The work in [8] provides location verification assisted by a moving obstacle for wireless sensor networks. A software-defined surveillance system with energy harvesting has been proposed in [9]. In [10], IoT architecture to detect the occurrence of both error and event in a forest environment has been presented.

The presented all works related to IoT and non IoT senor networks, motivate us to propose a work for the heterogeneous IoT-BLWSNs, where transmission of sensed events will be carried out layer to layer before sending to the

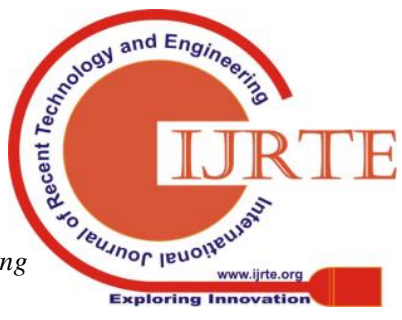


cloud. Hence, at the administrative end data from different layers will be in the prioritized order, which will help to reciprocate the sensed events in the fastest time duration to their concern authority.

\section{PROPOSED RESEARCH WORK}

An IoT based layered sensor network has been proposed in the Fig.1, which monitors a given large area surrounded by the forest. For easy to explain, we consider (1 x 1) km monitoring area partitioned into $100 \mathrm{~ms}$ of smaller sectional area based on the column basis, from the boundary line of forest (BLOF), we have named it as layer 1, layer 2, layer 3 so on till layer 10, towards the Microcontroller. The detection range of PIR sensors will define the number of rows presented in the system model, for the proposed work we consider range as $4 \mathrm{~m}$. Hence, two rows will be at $8 \mathrm{~m}$ apart and total number of rows required will be 125 .

The processing of data will carry out wirelessly layer to layer through the column basis in the presented system model of 125 rows and 10 columns. We consider, a fixed plank to the ground with plank stand having an intruder detection PIR sensor, smoke detection sensor, Omni directional antenna, humidity and temperature measurement sensor on top of it. One humidity and temperature sensor is present in each layer between two rows which communicates with the presented two humidity and temperature sensors placed on the plank as shown in the Fig.1.

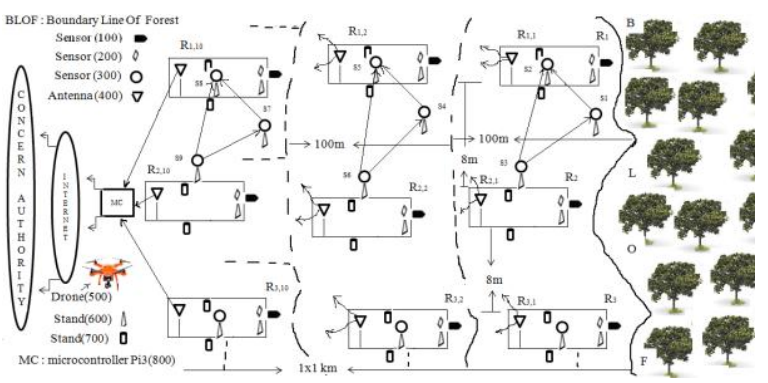

Figure.1

\section{CONCLUSION}

The proposed IOT based clustered WSN will be useful in the application where administrator will be at very far distance from deployed network such as village surrounded by forest. The presented work will also be useful in restricted campus area, where different events have to be detected with priority basis such as, in the hospital, ICU unit, Ward unit, and Emergency unit etc to be accessed by a common administrator. The given system model can also be used for monitoring the animal cage in the zoo campus. Further, the proposed network can be used for non restricted campus area where administrator wants to access the undesired detected events form any corner of the notified area.

\section{REFERENCES}

1. Nkwari, Patrick Kibambe Mashoko, et al. "Heterogeneous wireless network based on Wi-Fi and ZigBee for cattle monitoring." IST-Africa Conference, 2015. IEEE, 2015.

2. Du, Rong, et al. "Effective urban traffic monitoring by vehicular sensor networks." IEEE Transactions on Vehicular Technology 64.1 (2015): 273-286.

3. Wan, Liangtian, et al. "Distributed parameter estimation for mobile wireless sensor network based on cloud computing in battlefield surveillance system." IEEE Access 3 (2015): 1729-1739.

4. Postigo-Malaga, Mauricio, et al. "Vehicle location system and monitoring as a tool for citizen safety using wireless sensor network." ANDESCON, 2016 IEEE. IEEE, 2016.

5. Lee, Jin-Shyan, and Tsung-Yi Kao. "An Improved Three-Layer Low-Energy Adaptive Clustering Hierarchy for Wireless Sensor Networks." IEEE Internet of Things Journal 3.6 (2016): 951-958.

6. Naeem, Muhammad Kamran, Mohammad Patwary, and Mohamed Abdel-Maguid. "On Lifetime Maximisation of Heterogeneous Wireless Sensor Networks with Multi-Layer Realisation." Wireless Communications and Networking Conference (WCNC), 2017 IEEE. IEEE, 2017.

7. Wan, Shuo, et al. "To Smart City: Public Safety Network Design for Emergency." IEEE Access 6 (2018): 1451-1460.

8. Wu, Di, et al. "Location Verification Assisted by a Moving Obstacle for Wireless Sensor Networks." IEEE Internet of Things Journal 5.1 (2018): 322-335.

9. Ko, Haneul, and Sangheon Pack. "A Software-Defined Surveillance System with Energy Harvesting: Design and Performance Optimization." IEEE Internet of Things Journal 5.3 (2018): 1361-1369.

10. Nesa, Nashreen, Tania Ghosh, and Indrajit Banerjee. "Outlier detection in sensed data using statistical learning models for IoT." Wireless Communications and Networking Conference (WCNC), 2018 IEEE. IEEE, 2018.

11. Singh Mukesh Kumar, Govind Sharma, and Naren Naik. "Joint optimization of SINR and power allocation to relays in cluster-based wireless sensor networks." Signal Processing and Communications (SPCOM), 2014 International Conference on. IEEE, 2014 